Bull. Mater. Sci., Vol. 17, No. 6, November 1994, pp. 795-810. C Printed in India.

\title{
An electron diffraction study of quasicrystals in Ti-37 at\% Mn and Ti-24 at\% Mn-13 at\% Fe alloys
}

\author{
T A BHASKARAN, R V KRISHNAN and S RANGANATHAN* \\ Materials Science Division, National Aerospace Laboratories, Bangalore 560 017, India \\ *Centre for Advanced Study, Department of Metallurgy, Indian Institute of Science, \\ Bangalore 560012 , India
}

\begin{abstract}
Electron diffraction studies were carried out to establish the icosahedral phase formation in rapidly quenched $\mathrm{Ti}-37$ at\% $\mathrm{Mn}$ and $\mathrm{Ti}-24$ at\% $\mathrm{Mn}-13$ at\% $\mathrm{Fe}$ alloys. Distortions in the diffraction spots and diffuse intensities in the diffraction patterns were investigated. The existence of a rational approximant structure and a decagonal like phase are also reported.
\end{abstract}

Keywords. Quasicrystals; electron diffraction; titanium alloys.

\section{Introduction}

A remarkable discovery was made in/the year 1984 by Shechtman et al (1984) while studying the electron diffraction patterns of rapidly quenched Al-Mn alloys when they reported the formation of a solid phase, 1-2 $\mu \mathrm{m}$ in size, giving rise to sharp diffraction intensities like a single crystal but with a point group symmetry $m \overline{3} \overline{5}$ which is inconsistent with lattice translations.

The basic principles governing the synthesis of the quasicrystals can be simply understood as being based on the following criteria: (i) icosahedral crystal structure criterion, which derives its basis on the existence of crystalline phases possessing short range icosahedral order (Ramachandra Rao and Sastry 1985; Henley and Elser 1986), (ii) quantum structural diagram criterion based on quantum properties of the elements, and (iii) the phase diagram criterion-where a peritectic reaction is featured by the quasicrystal forming alloys.

While there are isolated examples of quasicrystals based on $\mathrm{Pd}$ and $\mathrm{Li}$ alloys, the vast majority of quasicrystals are aluminium-, magnesium- and titanium-based alloys (Ranganathan and Chattopadhyay 1991). Titanium based quasicrystals are attracting increasing attention, since their original discovery by Zhang et al (1985). Bhaskaran (1992) and Kelton (1994) have reviewed titanium quasicrystals as well as closely related rational approximant structures.

The occurrence of quasicrystals in Ti-Mn system is quite interesting since it does not follow any of the criteria discussed above (Kelton et al 1988). The Ti-Mn phase diagram does not show the $\mathrm{Ti}_{2} \mathrm{Mn}$ phase. The reported composition of I-phase in Ti-Mn alloy is near eutectic composition which is 37 at\% Mn. Kelton (1994) has shown the existence of higher order crystalline approximant structures in these alloys. From a comparison of the various models he pointed out the near agreement of the experimental results on arcs of diffuse intensity with the icosahedral glass model.

X-ray diffraction (XRD) studies on the rapidly quenched Ti-Mn alloys (Yavari 
and Verger-Gaugry 1988) showed close similarities to the icosahedral phase suggesting that its formation may be possible in this system. Kelton and co-workers (1988) were the first to successfully synthesize icosahedral quasicrystals in Ti-Mn alloys. The I-phase in Ti-Mn alloys assumes more significance because of the occurrence of arcs of diffuse scattering in electron diffraction patterns. It is believed that the position and intensity of these arcs will give valuable information about the atomic decoration (occupancy) of the quasicrystal lattice (Gibbons et al 1989). The stability of the quasicrystal in this alloy has been studied by Holzer et al (1989). It has been reported that the I-phase is stable below $1075 \mathrm{~K}$. Annealing at $1130 \mathrm{~K}$ for $30 \mathrm{~min}$ resulted in complete transformation of the I-phase. The transformation products did not correspond to any of the equilibrium phases. Further, a cubic phase with $a=13.44 \AA$ corresponding to the $1 / 1$ approximant structure has been found to occur (Kelton 1989).

In this paper the I-phase formation in rapidly quenched Ti-37 at $\% \mathrm{Mn}$ alloy and the occurrence of diffuse intensity are discussed. Transmission electron microscopy (TEM) studies were carried out to compare the characteristics of the quasicrystalline phase in this alloy to those of $\mathrm{Al}$ and $\mathrm{Mg}$ base quasicrystals and to determine whether any other competing phases are present. XRD has been used to characterize melt spun ribbons of this material and the vertex vectors of the I-phase determined for calculation of the quasilattice constant. TEM in conjunction with selected area electron diffraction (SAED) techniques have also been employed. A possible rational approximant structure closely connected to the I-phase has been identified. In addition, the shape distortion of the diffraction spots is reported. An icosahedral quasicrystal has also been observed in the rapidly quenched Ti-24 at\% Mn-13 at\% Fe alloy. The alloys used in this study have a silicon content of 2 to 3 at $\%$. It appears that the presence of silicon is essential for the formation of I-phase in these alloys (Kelton et al 1988).

\section{Experimental}

The melt spun ribbons of $\mathrm{Ti}-37$ at $\% \mathrm{Mn}$ and $\mathrm{Ti}-24$ at $\% \mathrm{Mn}-13$ at $\% \mathrm{Fe}$ were provided by Kelton. The details of melt spinning are given elsewhere (Kelton et al 1988). The ribbons were annealed in vacuum at 573 and $873 \mathrm{~K}$ respectively for $15 \mathrm{~min}$. Thin foils for electron microscopy were prepared in a Tenupol twin jet electropolishing unit using an electrolyte of $90 \%$ methanol and $10 \%$ sulphuric acid at $223 \mathrm{~K}$ and under a d.c. potential of $30 \mathrm{~V}$. Some samples were also prepared by ion thinning. The thin foils were observed in a Philips EM 300 and JEOL-200CX TEM operating at 100 and $200 \mathrm{kV}$ respectively. XRD was carried out in a Philips $\mathrm{X}$-ray diffractometer using copper $\mathrm{K}_{\alpha}$ radiation at $40 \mathrm{kV}$.

\section{Results and discussion}

\subsection{Quasicrystals in titanium-37 at\% manganese alloy}

3.1a Electron diffraction and microscopy: The XRD data of the as rapidly quenched alloy are given in table 1 . The indexing has been carried out according to Bancel et al (1985). The co-existence of the I-phase with other metastable phases is 
Table 1. X-ray diffraction results of rapidly quenched Ti-37 at $\% \mathrm{Mn}$ alloy.

\begin{tabular}{|c|c|c|c|c|}
\hline $2 \theta$ & $d(\AA)$ & Intensity & Phase & Index \\
\hline 35.8 & 2.501 & $\mathbf{w}$ & I & $(21 \overline{1} 001)$ \\
\hline $39 \cdot 8$ & $2 \cdot 264$ & $\mathrm{~m}$ & TiMn; $\beta \mathrm{Ti}(\mathrm{Mn})$ & $(320) ;(110)$ \\
\hline $40 \cdot 4$ & $2 \cdot 232$ & $\mathbf{s}$ & I & $(100000)$ \\
\hline $40 \cdot 8$ & 2.211 & $\mathrm{~m}$ & TiMn & (313) \\
\hline 41.6 & $2 \cdot 170$ & $\mathbf{m}$ & TiMn & (205) \\
\hline 42.5 & $2 \cdot 126$ & $\mathrm{~m}$ & I & $(110000)$ \\
\hline 43.45 & 2.082 & mw & I & $(220002)$ \\
\hline $58 \cdot 5$ & $1 \cdot 577$ & $w$ & $\beta-T i(M n)$ & $(200)$ \\
\hline $71 \cdot 8$ & $1 \cdot 314$ & w & I & $(2110 \overline{1} 1)$ \\
\hline $72 \cdot 2$ & 1.308 & $\mathbf{w}$ & $\beta-T i(M n)$ & $(211)$ \\
\hline $73 \cdot 35$ & $1 \cdot 290$ & w & I & $(101000)$ \\
\hline
\end{tabular}

w, weak; mw, medium weak; m, medium; s, strong.

indicated by the X-ray data. The competing crystalline phases are the $\beta$-Ti $(\mathrm{Mn})$ and TiMn. It is observed that the vertex vector (100000) is stronger than the edge vector (110000). This is also the case in Al-Mn alloys reported by Bancel et al (1985). However, the intensities are reversed in Mg-based quasicrystals (Mukhopadhyay 1989).

TEM studies revealed that a wide variation in the size of quasicrystals ranging from $0.07 \mu \mathrm{m}$ to $0.5 \mu \mathrm{m}$ exists. From figure 1 it is seen that the coarser quasicrystals co-exist with the $\beta$ titanium at the rim of the quasicrystals. The quasicrystals exhibit a very fine dendritic morphology and speckle contrast with each quasicrystal divided into a number of sectors. It was found that electron diffraction ring patterns obtained from the area containing very fine grains could be indexed in terms of the I-phase. The $d$ spacings of the rings in the electron diffraction pattern are in the $\tau$ ratio of 1.618 .

Figures 2a-c are SAED patterns taken with the electron beam parallel to the 5-fold, 3-fold and 2-fold axis directions, respectively, in the quasicrystalline phase, confirming the $m \overline{\mathbf{3}} \overline{\mathbf{5}}$ symmetry. In addition the [ $\bar{\tau} 10]$ zone axis pattern was also obtained (figure 2d). Dark field image of the quasicrystal showed that the entire quasicrystal is illuminated suggesting that all the sectors inside the quasicrystal possess the same orientation.

The variation in the size of the quasicrystal observed is in accordance with the study of Kelton et al (1988). The internal structure of the quasicrystal is very similar to that of the I-phase reported in $\mathrm{Mg}_{32}(\mathrm{Al}-\mathrm{Zn}-\mathrm{Cu})_{48}$ and in many other Al-Mn alloys (Mukhopadhyay et al 1987a). However, in the present case the boundaries between the quasicrystals are not faceted. Instead, they are irregular (figure 1). The speckle contrast observed can be interpreted on the basis of the results of Knowles and Stobbs (1987) who carried out detailed dark field imaging of the quasicrystals to understand the origin of the speckle contrast and traced it to the chemical inhomogeneities present in the quasicrystalline phase. It has been pointed out by Kim et al (1990) that various factors such as micrograin structure, topological defects like phasons and phonons, chemical inhomogeneity, local periodicity and decagonal phase may be sources for the observed deviations from the icosahedral symmetry. 


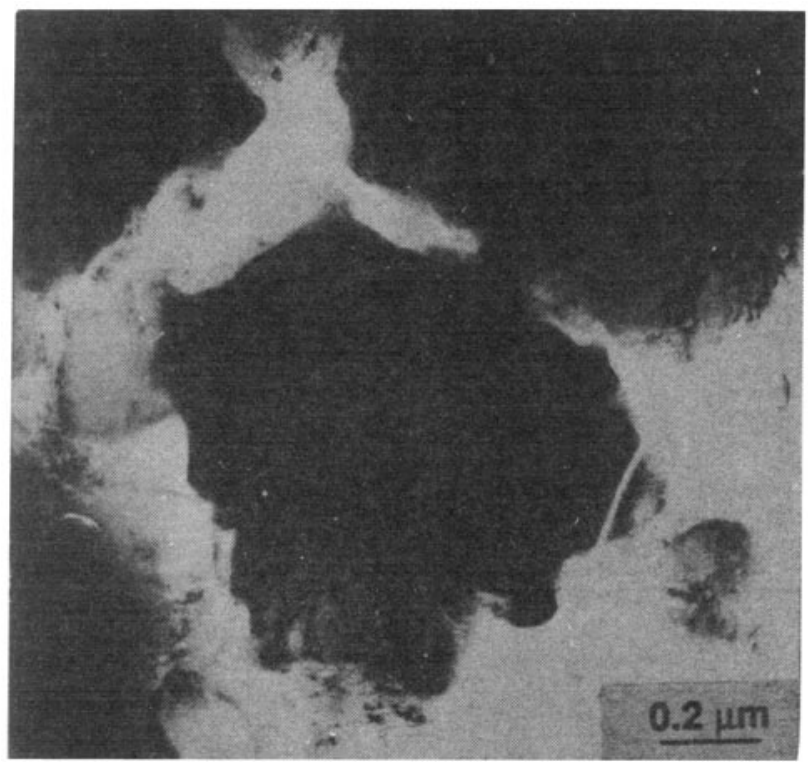

Figure 1. TEM of melt spun Ti-37\% Mn alloy showing icosahedral quasicrystals exhibiting speckle contrast with retained $\beta$ surrounding the rim of the I-phase.

$3.1 b$ Quasilattice constant: The quasilattice constant has been defined as the edge length $\left(a_{R}\right)$ of the rhombohedra of the three dimensional Penrose tiling (Elser and Henley 1985). The quasilattice constant is calculated as

$$
a_{R}=\left(\tau^{3} / 2\right) \cdot d_{100000}
$$

Substituting the value of $d_{100000}=2.232 \AA$ in the above expression the quasilattice constant $\left(a_{R}\right)$ is equal to $4.73 \AA$. This value is different from that for the $\mathbf{M g}$ base alloys and closer to that in A1-Mn quasicrystals. While $a_{R}=5.15 \AA$ for $\operatorname{Mg}_{32}(\mathrm{AlZn})_{48}$ (Mukhopadhyay 1989), it is reported to be $4.60 \AA$ for $\alpha(\mathrm{Al}-\mathrm{Mn}-\mathrm{Si})$ (Henley and Elser 1986). The results obtained in the present case are also close to those reported for $\mathrm{Ti}_{2} \mathrm{Ni}$ by Dunlap et al (1988) where $a_{R}=4.76 \AA$.

3.1c Distortions in the shape of diffraction spots: An enlarged 5-fold diffraction pattern of the quasicrystal is shown in figure 3. Subtle variations in peak intensity and shape of the spots exist compared to the ideal quasicrystalline diffraction pattern. It is observed that the inner ring of diffraction spots is weak and pentagons are more intense while the reverse is the case in Al-Mn quasicrystals. Further, it is noticed that the innermost diffraction spots close to the transmitted spot are not circular but triangular in shape. The vertices of the triangle are pointing towards the central spot. The shape of the diffraction spot changes from triangular to circular at higher $\vec{g}$ values. The second generation spots are neither perfectly triangular nor circular. The diffraction spots closer to the transmitted spot in the 2-fold pattern are also not perfectly circular. It is also noticed that the diffraction spots comprising the inner pentagons are triangular in shape. The circumferential deviation of the 

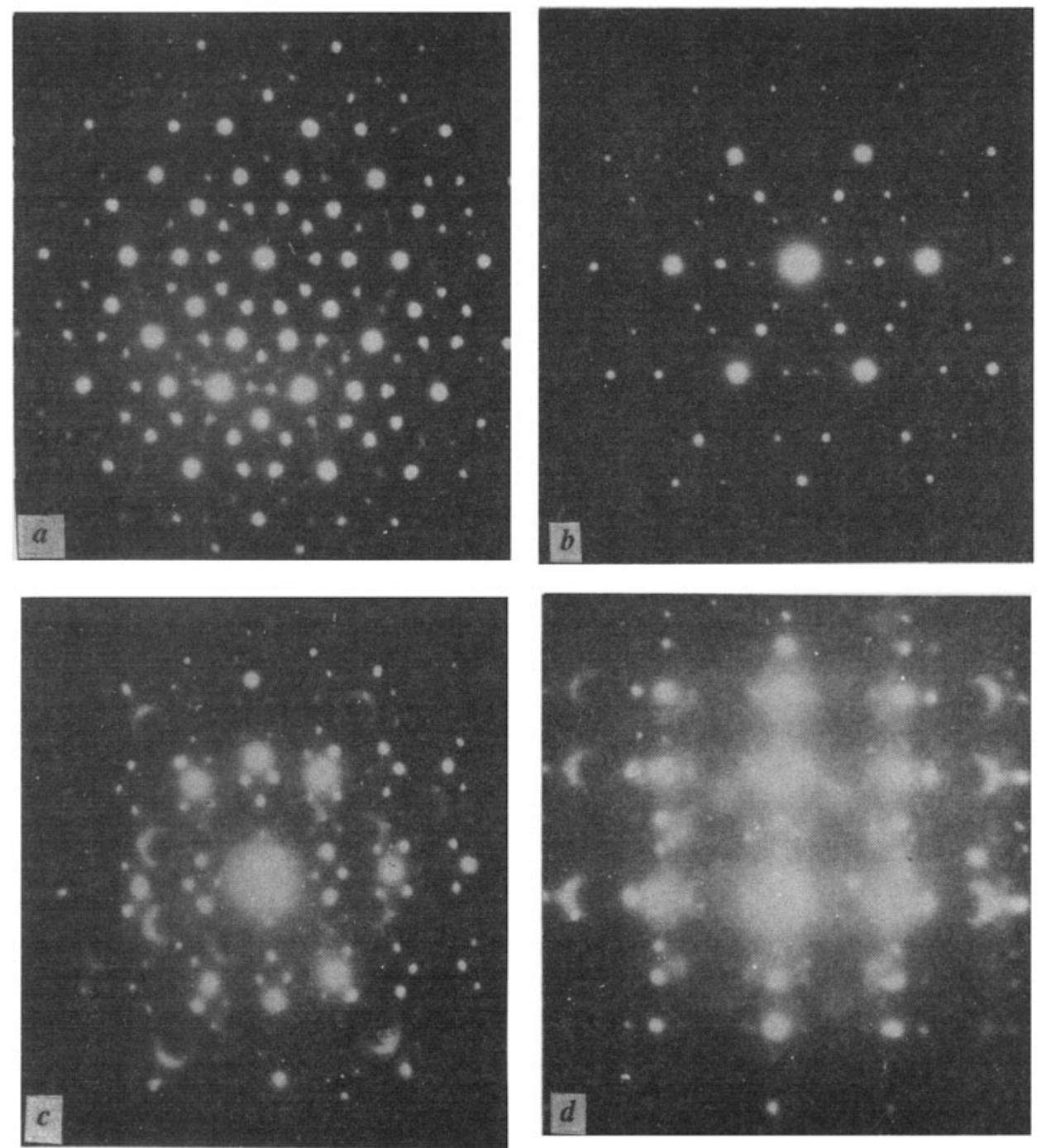

Figure 2. a. F-Fold b. 3fold, c. 2.foid and d. [F.0] SAEn pattern from the I-phase.

diffraction spots can be noticed in the 5-fold pattern of the sample annealed at $573 \mathrm{~K}$ (figure 4 ).

Observations of the shape distortion of diffraction spots have been reported in $\mathrm{Al}_{78} \mathrm{Cr}_{17} \mathrm{Ru}_{5}$ (Bancel and Heiney 1986), $\mathrm{Al}-\mathrm{Li}-\mathrm{Cu}$ ( $\mathrm{Yu}$ et al 1987) and $\mathrm{Mg}-\mathrm{Al}-\mathrm{Zn}$ alloys (Chandra and Suryanarayana 1988). Various models and theories have been proposed to explain the observed shape distortion of the diffraction spots. Lubensky et al (1986) attributed these distortions to the presence of disorder in the quenched state of the quasicrystal. Socolar and Wright (1987) pointed out that the peak broadening in XRD and distortions of the spots in SAED patterns are the result of the anisotropic strain in the phason field of the icosahedral quasicrystalline phase. Zhang and Kuo (1987) explained these on the basis of local translational 


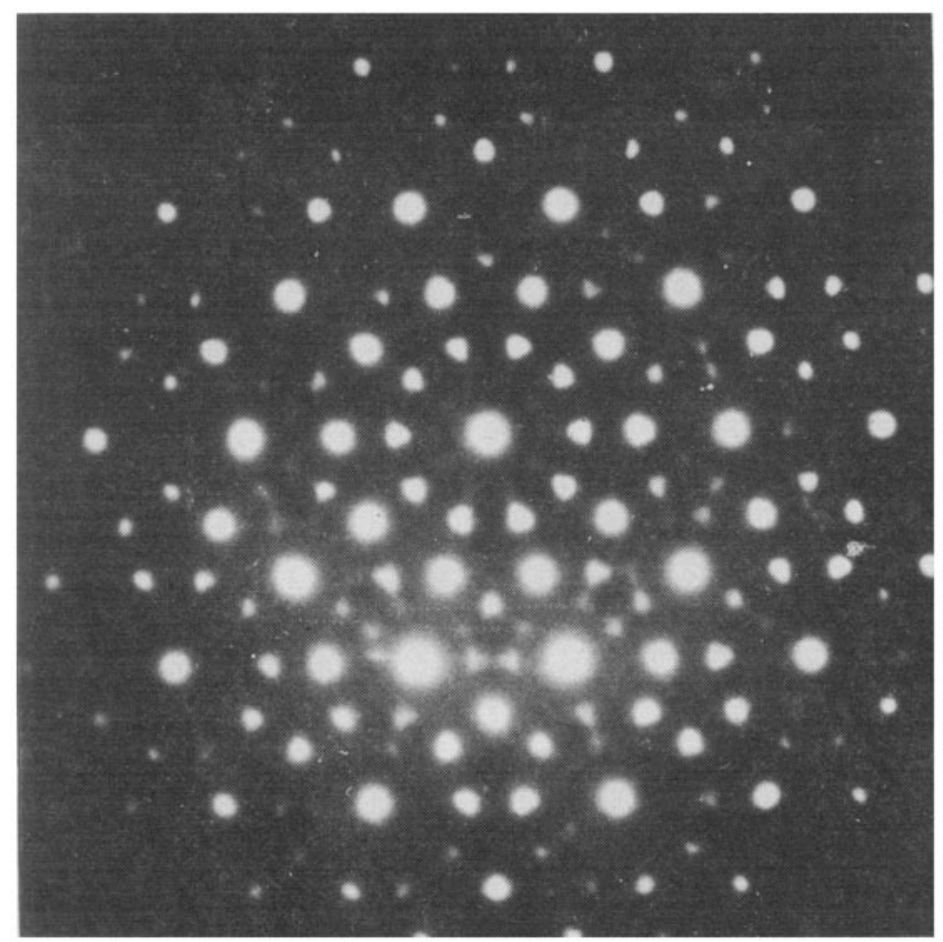

Figure 3. Enlarged 5-fold SAED pattern in Ti-37\% Mn alloy showing triangularly distorted inner spots.

order occurring on several two-fold directions normal to the five-fold axis in different subgrains of the quasicrystal. An explanation based on rational approximant structure has been advanced for the observed distortions without taking support of the phason strain concept (Mukhopadhyay 1989). In our study due to the limited observations it has not been possible to propose any model. However, it is possible that any one or combinations of the models proposed earlier could explain the shape distortions of the diffraction spots.

3.1d Diffuse intensity: Arcs of diffuse intensity have been observed in the electron diffraction patterns of $\mathrm{Ti}-37 \% \mathrm{Mn}$ in rapidly quenched and annealed conditions (Gibbons et al 1989; Bhaskaran et al 1993). In Ti-Mn alloy the diffuse intensity occurs in the quenched state itself (figures $2 \mathrm{c}$ and $\mathrm{d}$ ). This is to be compared to the Al-Mn alloy where it has been reported to manifest only after annealing the quasicrystal (Chattopadhyay and Mukhopadhyay 1987; Mukhopadhyay et al 1987b). The intensity of the arcs is stronger in Ti-Mn alloy (Bhaskaran et al 1993; Gibbons et al 1987) compared to the hitherto reported observations in Ti-Fe (Dong et al 1987), Mg-Al-Zn (Mukhopadhyay et al 1987a), Al-Mn (Chattopadhyay and Mukhopadhyay 1987; Mukhopadhyay et al 1987b) and Al-Li-Cu alloys (Goldman et al 1988). Mandal et al (1991) observed that additions of silicon up to $6 \%$ to $\mathrm{Ti}_{2} \mathrm{Fe}$ resulted in I-phase. They also observed arcs of diffuse intensities similar to Ti-Mn quasicrystal. 


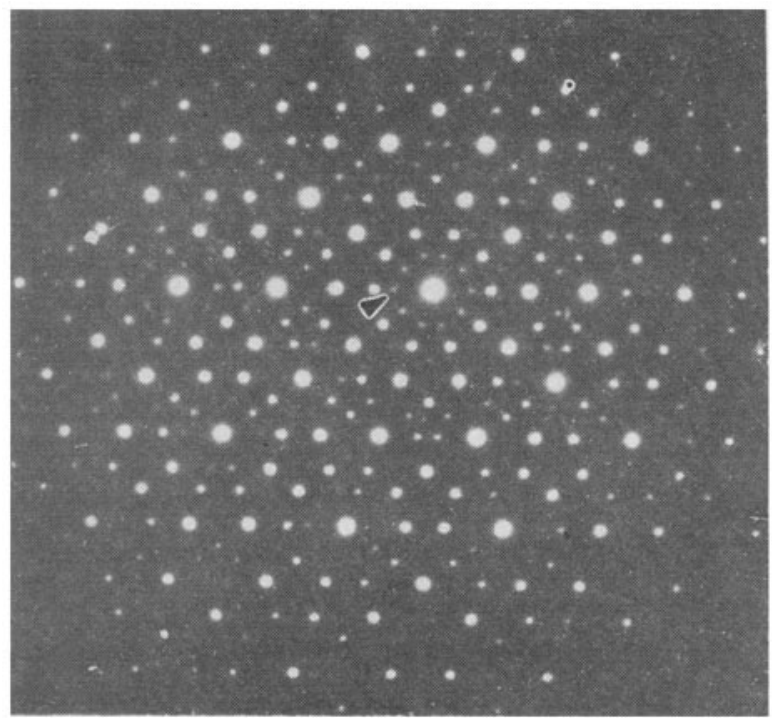

Figure 4. 5-Fold SAED pattern of Ti-37\% $\mathrm{Mn}$ alloy after annealing at $573 \mathrm{~K}$ showing misalignment of spots.

In our earlier work (Bhaskaran et al 1993), annealing experiments were carried out to study the possible changes in the arcs of diffuse intensity. The diffuse intensities present in the 2-fold and [ $\bar{\tau} 10]$ patterns of the as quenched alloy were compared to those of the annealed sample. The results indicated that the arcs of diffuse intensities have given place to sharp diffraction spots upon annealing. It was noticed that the diffuse intensities together with the spots along the odd parity directions are quasiperiodic following $\tau$ inflation. The spots alone follow $\tau^{3}$ scaling.

Figures 5a-f illustrate the results of tilting experiments carried out about a three-fold diffraction pattern. Even at a small tilt of $2^{\circ}$, one can notice the occurrence of triangular shape intensities and these are well developed at a tilt of $6^{\circ}$. Further tilting to $10.5^{\circ}$ from, the three-fold orientation has resulted in $(\bar{\tau} 10)$ pattern. At a tilt of $27^{\circ}$ very strong arcs are noticed. The results of tilting from the 5-fold orientation about one of the 2 -fold axis are shown in figures $6 a-d$. Arcs of diffuse intensities are noticed at a tilt of $4^{\circ}$ and they are stronger at a tilt of $20^{\circ}$.

The existence of diffuse intensity in the electron diffraction pattern of Al-Mn quasicrystal was first reported by Mukhopadhyay et al (1987b) after annealing the quasicrystal. It was suggested that the diffuse intensities in Al-Mn quasicrystal are evidence for the short range order taking place before the onset of long range order. Henley (1988) showed that the locations of diffuse intensity at $\tau$ ratio on odd parity directions can be correlated to the existence of a face centered cubic quasicrystal. Experimental evidence for the existence of fcc type quasicrystal was obtained in Al-Cu-Fe system by Ebalard and Spaepen (1989). The 2-fold pattern of the I-phase in this alloy showed additional diffraction spots compared to the simple $p$ type I-phase normally observed in Al-Mn alloys. These authors have proposed the existence of a fcc type I-phase to explain the presence of additional diffraction spots. Mukhopadhyay et al (1989) pointed out that the simple icosahedral 

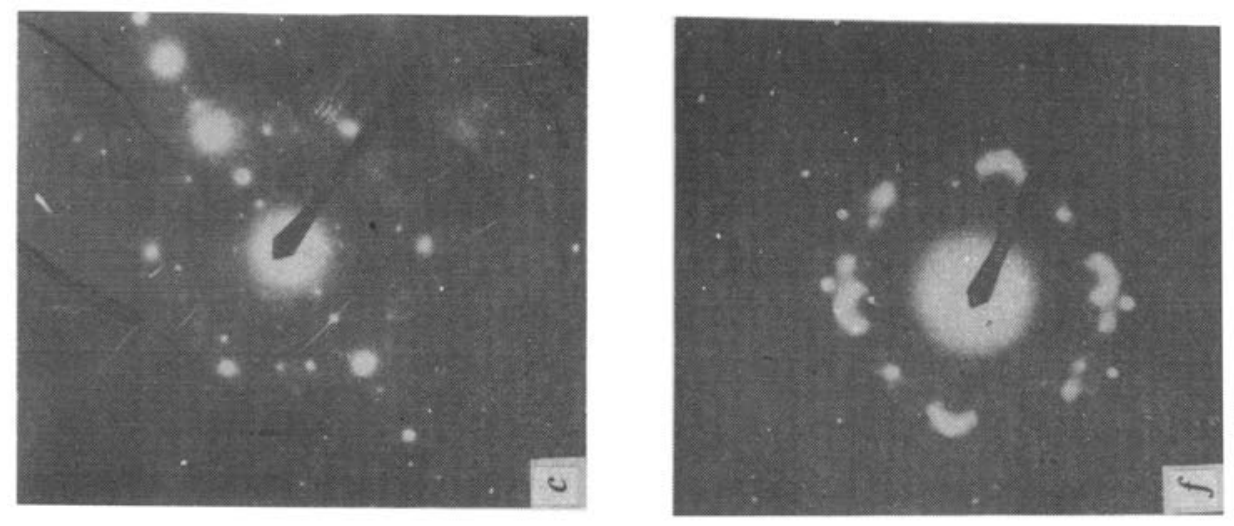

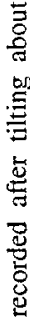
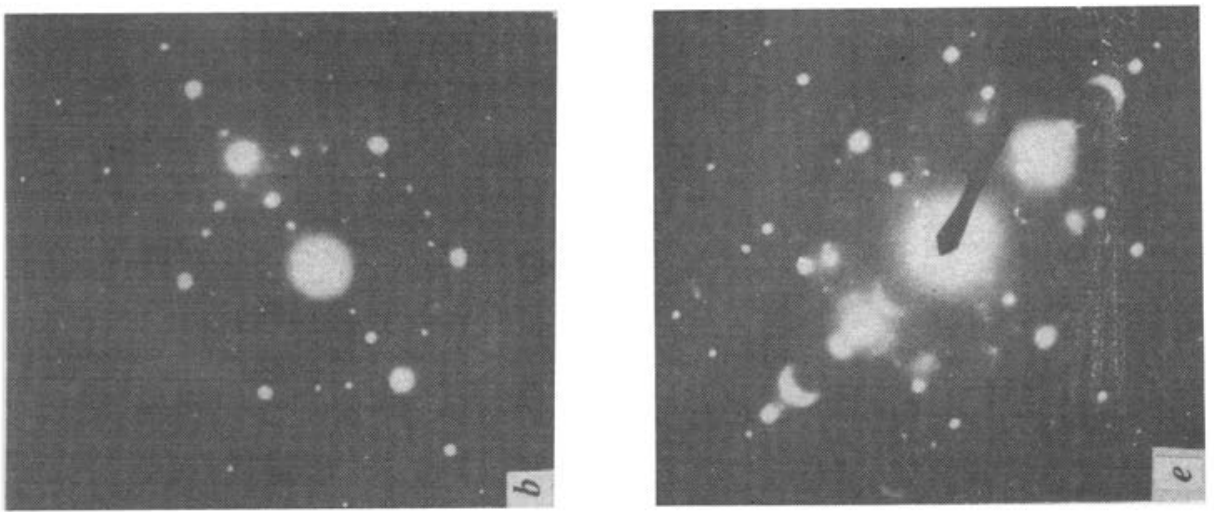

总

흘

茫

8

$\stackrel{0}{=}$

$\Xi$ in

冚

官.

io

$\dot{\circ} \dot{4}$

E

萢品

요
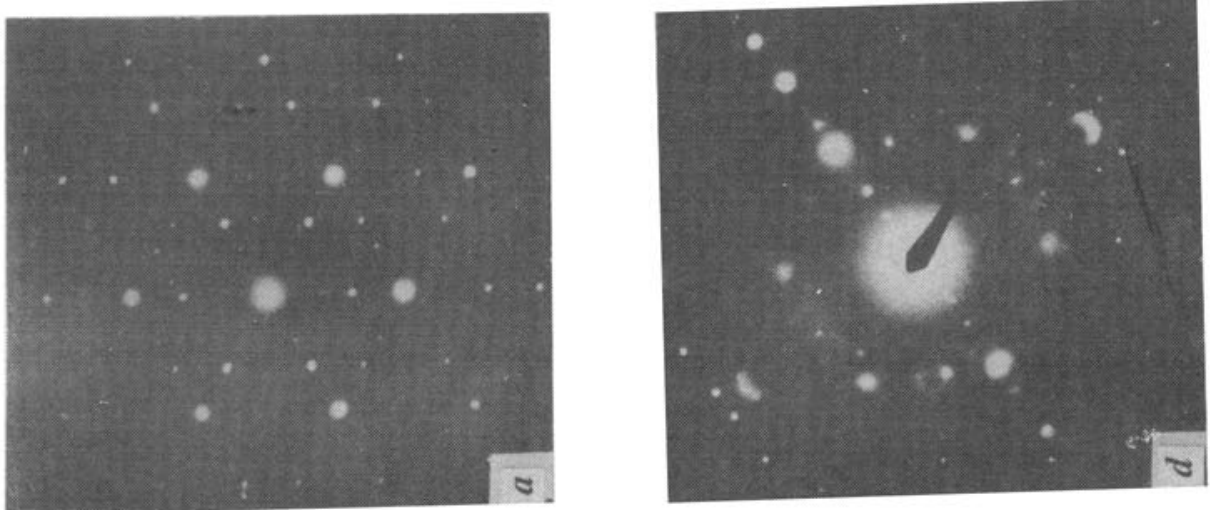

它

믕

if

드응

में

in

눙

范 

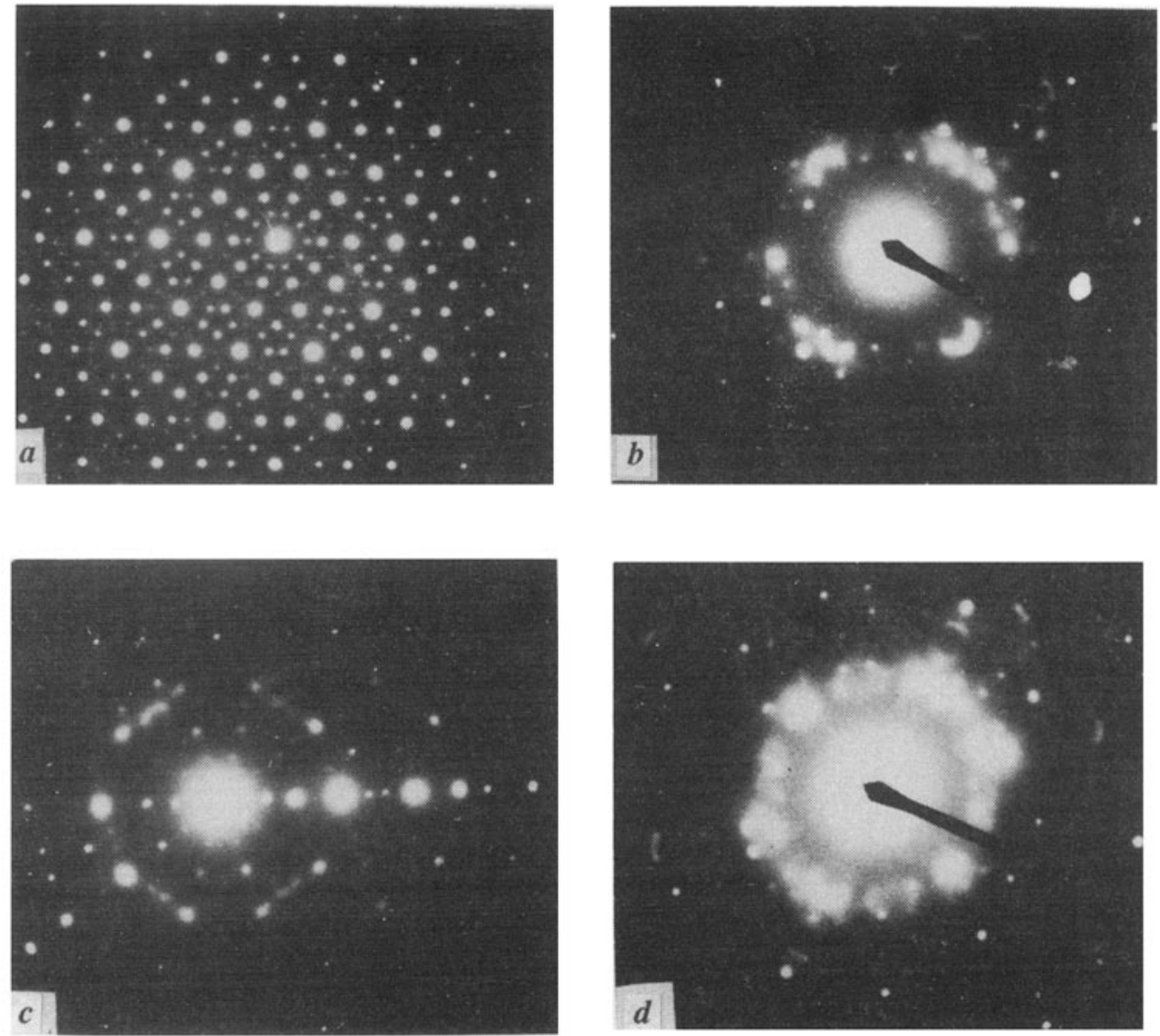

Figure 6. a. 5-Fold SAED pattern of the I-phase in Ti-37\% Mn alloy. Patterns recorded after tilting about one of the 2 -fold axis by b. $4^{\circ}$, c. $10^{\circ}$ and d. $20^{\circ}$.

to face centered icosahedral ordering transformation takes place in Al-10\% $\mathrm{Mn}$ alloy when annealed at a higher temperature and the superlattice spots appear in place of diffuse intensity. Tsai et al (1990) reported that the I-phase formed in $\mathrm{Al}-\mathrm{Mn}-\mathrm{Pd}$ is a superstructure of the simple icosahedral quasicrystal similar to the fcc type icosahedral phase reported in $\mathrm{Al}-\mathrm{Cu}-\mathrm{Fe}$ system. From the above studies it is evident that the occurrence of diffuse intensity in the diffraction patterns have indicated the existence of I-phase other than the primitive type.

The results of Gibbons et al (1989) in Ti-Mn indicated that the experimentally observed arcs of diffuse intensity could be explained based on the icosahedral-glass model than on a model based on the transition state theory. The present observations of arcs of diffuse intensity are to be viewed in the context of the above mentioned studies.

3.1e Rational approximant structure: Figures 7a-b show the metastable crystalline phases present in the rapidly quenched alloy. In figure $7 \mathrm{a}$ it is noticed that some of the grains exhibit a faulted structure. Closer to this phase a strongly diffracting phase is also noticed in figure $7 \mathrm{~b}$. The selected area diffraction pattern from the 


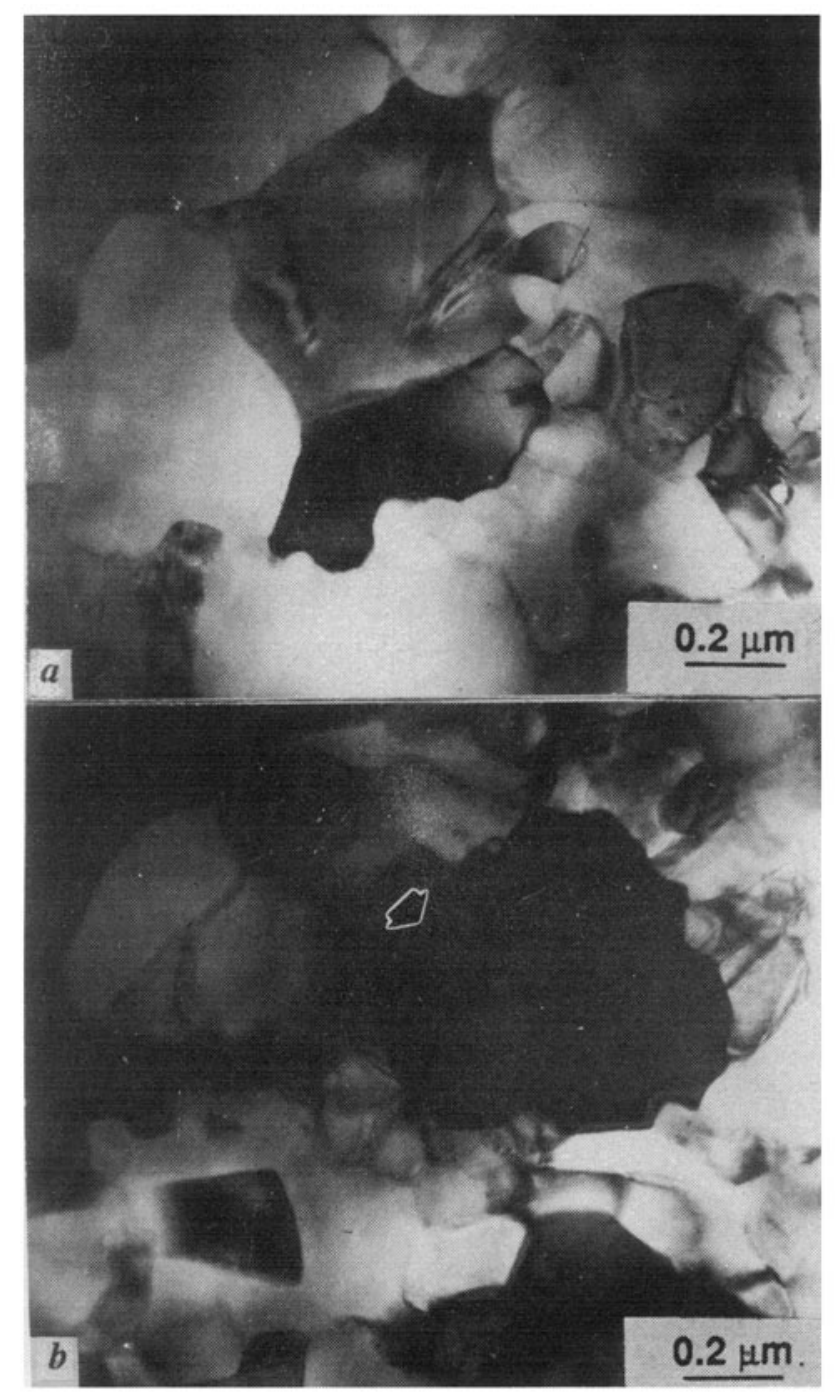

Figure 7. TEM of the metastable phases present in Ti-37\% Mn alloy along with the I-phase. In a. faults are seen and b. shows the phase (marked by arrow) giving a pseudo 5-fold diffraction pattern.

strongly diffracting phase is shown in figure 8. The diffraction pattern corresponds to the pseudo-five-fold symmetry. The inner spots marked by arrows in the diffraction pattern are approximately in the $\tau$ ratio. This pattern could be indexed in terms of a bcc structure with a lattice parameter of $21.7 \AA$.

Elser and Henley (1985) showed that the $\alpha$-(AlMnSi) crystal structure is closely related to that of the icosahedral quasicrystal in Al-Mn-Si alloys. By modifying the projection construction of the three-dimensional Penrose tilings adopted to generate the quasiperiodic structure, they were able to generate periodic structures and correlate the cubic $\alpha$-(AlMnSi) structure as an approximant structure of the 


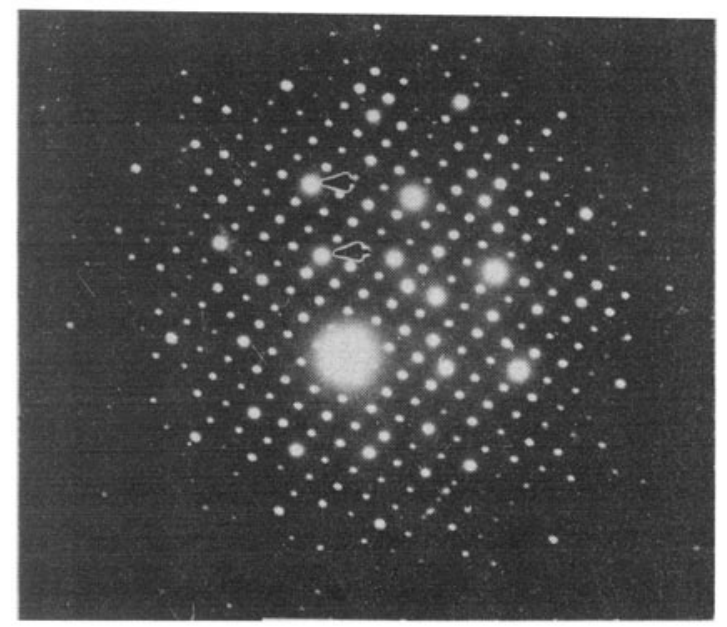

Figure 8. SAED pattern from the metastable phase shown in figure $7 \mathbf{b}$ exhibiting pseudo S-fold symmetry.

icosahedral quasicrystal I-(AlMnSi). The lattice constant $\left(a_{c}\right)$ of the approximant cubic structure is related to the quasicrystalline constant $a_{R}$.

For the $1 / 1$ rational approximant structure, the cubic lattice constant is given by

$$
a_{c}=[X] \cdot \tau,
$$

where

$$
X=\left[(2+2 / \sqrt{5})^{1 / 2} \cdot a_{R}\right]
$$

For the $2 / 1$ approximant structure the lattice constant is given by

$$
a_{e}=[X] \cdot \tau^{2} \text {. }
$$

Substituting $a_{R}=4.73 \AA$ for the Ti-Mn quasicrystals, the $1 / 1$ approximant structure has a cubic lattice constant $a_{c}=13.02 \AA$. Similarly for the $2 / 1$ approximant structure $a_{c}=21.07 \AA$. This suggests that the bcc phase observed in figure 8 is a $2 / 1$ rational approximant to the quasicrystalline phase.

Holzer et al (1989) have observed a bcc phase with $a=13.44 \AA$ after annealing the icosahedral quasicrystals in Ti-Mn alloy at $1275 \mathrm{~K}$ for $30 \mathrm{~min}$. In this alloy, however, the cubic phase observed by them may correspond to a $1 / 1$ approximant. Similarly, Dong et al (1987) have also reported a phase designated as $\alpha$ with $a=13 \cdot 6 \AA$.

3.1f Decagonal like phase: In addition to the observations reported above, it was often noticed that very fine crystallites existed surrounding the icosahedral phase in the rapidly quenched alloy. These fine crystals showed striated contrast (figure 9). The SAED patterns from these phases seem to be very complex (figures $10 \mathrm{a}-\mathrm{c}$ ). The intense spots in the diffraction pattern are found to be in the $\tau$ ratio. In these diffraction patterns, less intense spots from a crystalline phase are seen to be 


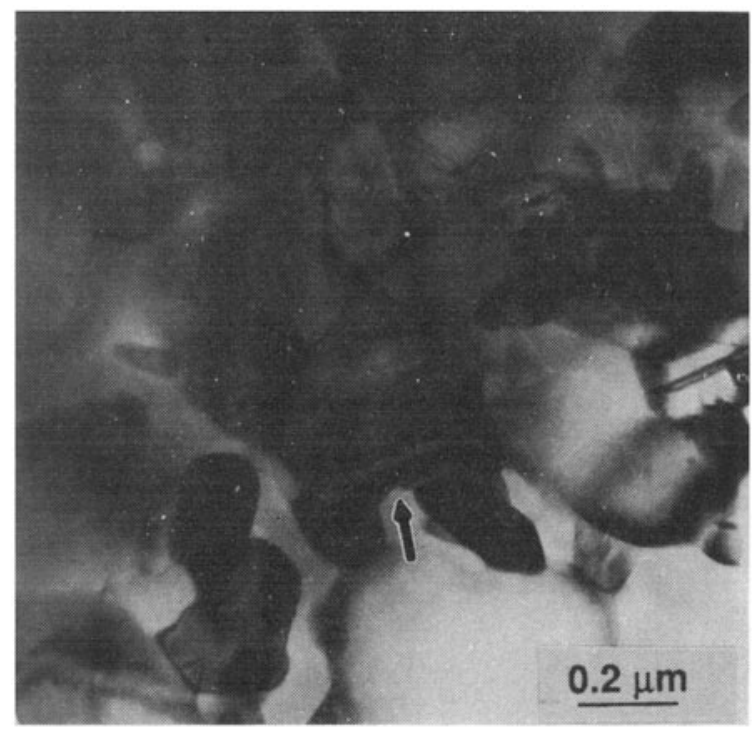

Figure 9. TEM showing decagonal like phase with striated contrast.

superimposed. The spots in figure 10a could be indexed as the $(7 \overline{2} \overline{5} 3)$ zone of a hexagonal phase. The lattice parameters of this hcp phase are $a=8.0 \AA$ and $c=15.7 \AA$ in conformity with the results of Levine et al (1992). Further, it was often found that the patterns exhibited extensive streaking. On tilting, the streaking is found to consist of many weaker diffraction spots. The diffraction pattern shown in figure $10 \mathrm{c}$ corresponds to the 3 -fold pattern. While the spots along one of the 2 -fold axis is in the $\tau$ ratio, the spots in the other two-fold directions are nearly commensurate. Further, one can notice that there is a shift in the spot positions. No ten-fold symmetry was observed. These patterns suggest that a decagonal like phase exists in Ti-Mn alloys following appropriate processing.

\subsection{Quasicrystal in Ti-24 at\% Mn-13 at\% Fe alloy}

The XRD data of the rapidly quenched alloy is shown in table 2 . The diffraction peaks correspond to the I-phase and $\beta \mathrm{Ti}(\mathrm{Mn})$. It is observed that the edge vector is stronger than the vertex vector in Ti-Mn-Fe quasicrystal. The I-phase in this alloy (figure 11) also exhibits speckle contrast similar to that in the $\mathrm{Ti}-37$ at\% $\mathrm{Mn}$ alloy. The grain boundaries of the I-phase are found to be decorated by very fine particles. The size of the quasicrystal seems to be bigger than that in the binary alloy suggesting that iron helps stabilize the I-phase.

The SAED patterns corresponding the 5-fold, 3-fold and 2-fold orientations are shown in figures 12 a-c. In the 5 -fold pattern it is seen that the inner spots are now more or less circular. However, the arcs of diffuse intensity in the 2-fold pattern are more intense in this alloy even in the quenched condition. They lie on the odd parity directions in the 2 -fold patterns. Additional diffraction patterns about the $[\bar{\tau} 10]$ axis and an intermediate zone axis, shown in figures $12 \mathrm{~d}$ and e exhibit strong arcs of diffuse intensity. 


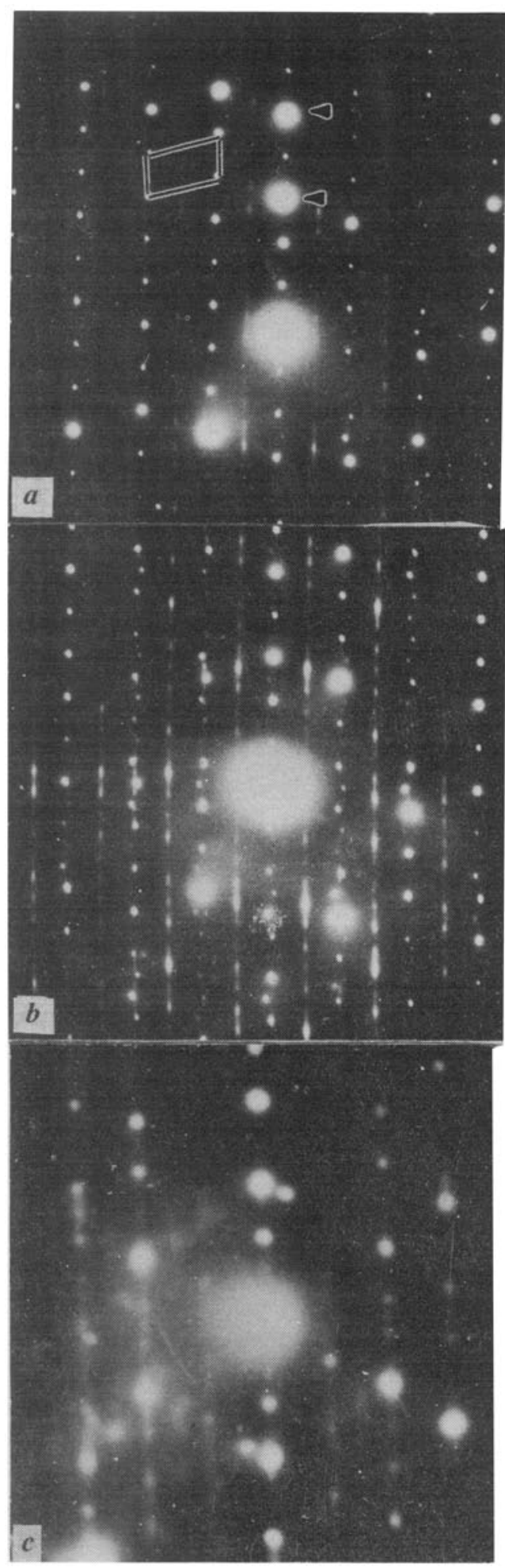

Figure 10. a-c. SAED patterns from the decagonal like phase showing quasiperiodicity along one direction. 
Table 2. X-ray diffraction results of $\mathrm{Ti}-24 \% \mathrm{Mn}-13 \% \mathrm{Fe}$ in as quenched condition.

\begin{tabular}{lcccl}
\hline $2 \theta$ & $d(\AA)$ & Intensity & Phase & Index \\
\hline 25.35 & 3.512 & w & I & $(1110 \overline{1} 0)$ \\
28.7 & 3.108 & w & I & $(2210 \overline{0} 0)$ \\
40.3 & 2.237 & m & I & $(100000)$ \\
40.95 & 2.203 & w & BTi; TiMn & $(110) ;(320)$ \\
42.45 & 2.129 & s & I & $(110000)$ \\
71.60 & 1.317 & w & I & $(2110 \overline{1} 1)$ \\
\hline
\end{tabular}

w, weak; m, medium; s, strong.

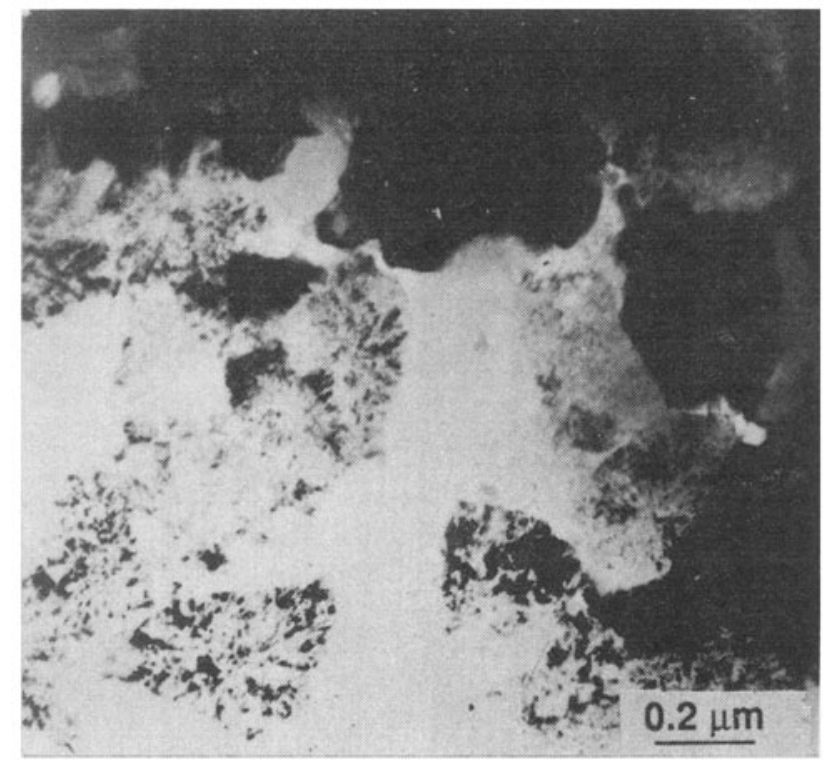

Figure 11. TEM of the I-phase in Ti-24\% $\mathrm{Mn}-13 \% \mathrm{Fe}$ alloy showing speckle contrast.

Following the method described in $\S 3.1 \mathrm{~b}$, the quasilattice constant for this alloy is calculated as $a=4.74 \AA$. This is not very different from the quasilattice constant for the I-phase in $\mathrm{Ti}-37$ at\% $\mathrm{Mn}$ alloy.

\section{Conclusions}

The formation of the I-phase in Ti-37\% $\mathrm{Mn}$ and Ti-24\% $\mathrm{Mn}-13 \% \mathrm{Fe}$ have been followed by TEM studies. The shape distortions of the diffraction spots observed in this study are similar to those reported in the I-phase of other alloys. Arcs of diffuse intensity observed in the quenched alloy have given rise to a number of sharp but faint diffraction spots after annealing. These are compared with the arcs of diffuse intensity reported in $\mathrm{Al}-\mathrm{Mn}, \mathrm{Al}-\mathrm{Cu}-\mathrm{Fe}$ and other alloys. In addition to 


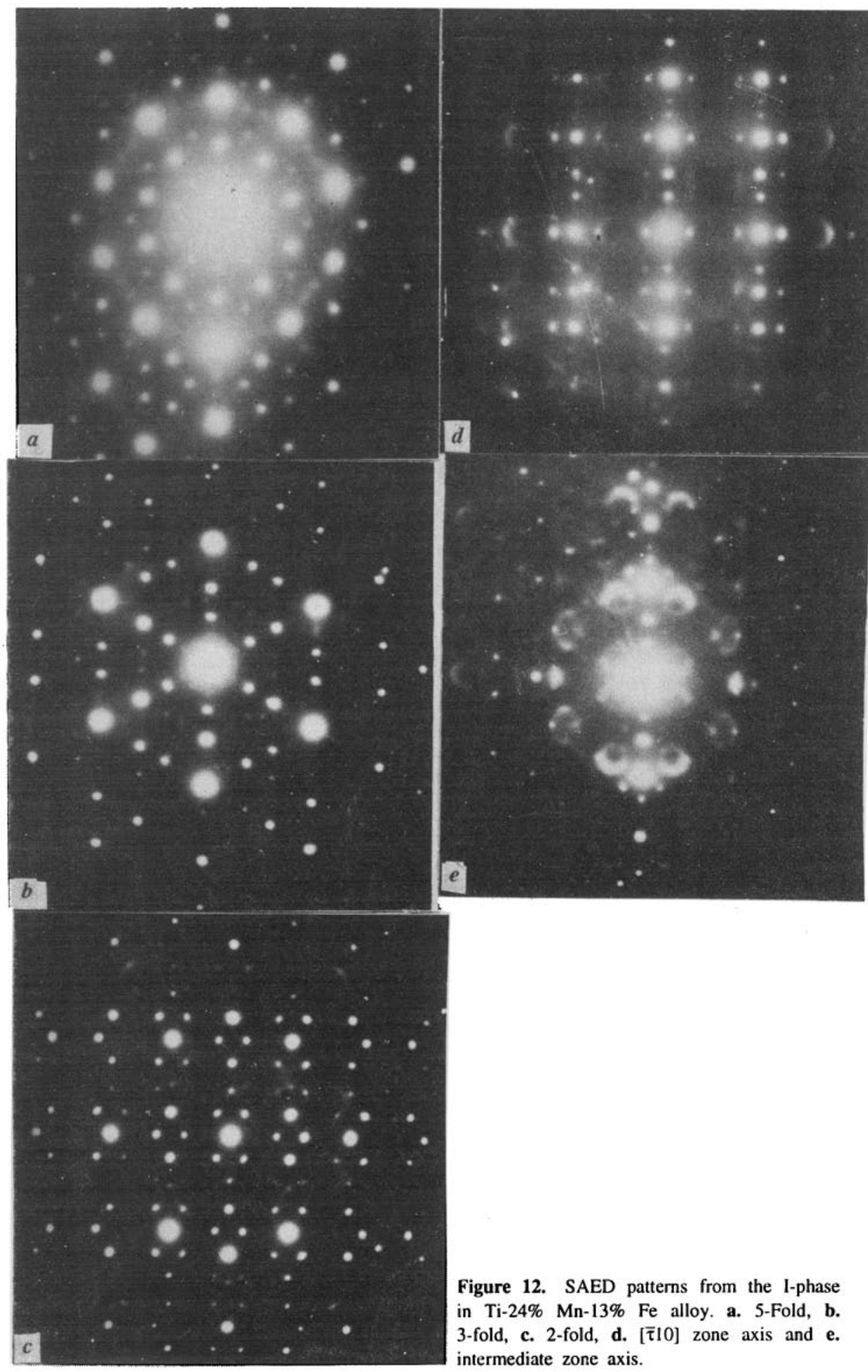


the I-phase, a rational approximant structure and another striated phase have also been observed in the $\mathrm{Ti}-37 \% \mathrm{Mn}$ in the quenched state.

\section{Acknowledgements}

The authors wish to thank $\operatorname{Dr} \mathbf{N}$ K Mukhopadhyay for very useful discussions. TAB and RVK thank Dr K N Raju, Director and Dr A K Singh, Head, Materials Science Division, National Aerospace Laboratories, for the keen interest and encouragement to this study. They wish to thank Prof. K F Kelton, Washington University, Missouri, USA for providing the melt spun Ti-Mn ribbons. They also acknowledge $\operatorname{Dr} \mathrm{G} N$ Subbanna for the help in some of the TEM work.

\section{References}

Bancel P A and Heiney P A 1986 J. Phys. 47 C3-341

Bancel P A, Heiney P A, Stephen P W, Goldman A I and Horn P M 1985 Phys. Rev. Lett. 542422 Bhaskaran T A 1992 Int. J. Rapid Solid. 7127

Bhaskaran T A, Krishnan R V, Ranganathan S and Kelton K F 1993 Proc. Indo-US Workshop on metastable microstructures, Goa, India, (eds) D Banerjee and L A Jacobson (New Delhi: Oxford and IBH Pub.).p. 93

Chandra S and Suryanarayana C 1988 Philos. Mag. B58 185

Chattopadhyay K and Mukhopadhyay N K 1987 Mater. Sci. Forum 22-24 639

Dong .C, Kuo K H and Chattopadhyay K 1987 Mater. Sci. Forum 22-24 555

Dunlap R A, McHenry M E, O'Handley R C and Chatterjee R 1988 Phys. Rev. B37 8484

Ebalard S and Spaepen F $1989 J$. Mater. Res. 439

Elser V and Henley C L 1985 Phys. Rev. Lett. 532883

.Gibbons P C, Kelton K F, Levine L E and Philips R B 1989 Philos. Mag. B59 593

Goldman A I, Guryan A C, Stephens P W, Parsey J M, Aeppli G Jr, Chen H S and Gayle F W 1988 Phy. Rev. Lett. 611962

Henley C L 1988 Philos. Mag. Lett. 5887

Henley C L and Elser V 1986 Philos. Mag. B53 L59

Holzer J C, Kelton K F, Levine L E and Gibbons P C 1989 Scr. Metall. 23691

Kelton K F 1989 Phase Trans. 16 \& 17367

Kelton K F 1994 in Advances in physical metallurgy (eds) S Banerjee and R Ramanujan (New York: Gordon and Breach Publishers) (in press)

Kelton K F, Gibbons P C and Sabes P N 1988 Phys, Rev. B38 7810

Kim D H, Hutchison J L and Cantor B 1990 Philos. Mag. A61 167

Knowles K M and Stobbs W M 1987 J. Microsc, 146267

Levine L, Holzer J C, Gibbons P C and Kelton K F 1992 Philos. Mag. B65 435

Lubensky T C, Socolar I E S, Steinhardt P J, Bancel P A and Heiney P A 1986 Phys. Rev. Lett. 571440 Mandal P, Tiwari R S and Srivastava O N 199I Philos. Mag. A63 617

Mukhopadhyay N K 1989 Some aspects of synthesis, structure and stability of quasicrystals, Ph. D Thesis, Indian Institute of Science, Bangalore

Mukhopadhyay N K. Thangaraj N, Chattopadhyay K and Ranganathan S 1987a J. Mater. Res. 2299

Mukhopadhyay N K, Ranganathan S and Chattopadhyay K 1987b Philos. Mag. Lett. 56121

Mukhopadhyay N K, Ranganathan S and Chattopadhyay K 1989 Philos. Mag. Lett. 60207

Ramachandra Rao P and Sastry G V S 1985 Pramana-J. Phys. 25 L225

Ranganathan S and Chattopadhyay K 1991 Ann. Rev. Mater. Sci. 21437

Schaefer R J 1986 Scr. Metall. 201187

Shechtman D, Blech I, Gratias D and Cahn J W 1984 Phys. Rev. Lett. 531951

Socolar I E S and Wright D C 1987 Phys. Rev. Lett. 57221

Tsai A P, Inoue A, Yokoyama Y and Masumoto T 1990 Philos. Mag. Lett. 619

Yavari A R and Verger-Gaugry I L 1988 J. Mater. Sci. 233383

Yu N, Portier R, Gratias D, Yu-Zhang K and Bigot J 1987 Mater. Sci. Forum. 22579

Zhang $Z$ and Kuo K H 1987 J. Microsc. 146313

Zhang Z, Ye H Q and Kuo K H 1985 Philos. Mag. A52 49 